\title{
LINGUISTIQUE
}

\author{
BIRGIT APFELBAUM
}

Université de Hildesheim

\section{LA GESTION DU COUPLE QUESTION-RÉPONSE (Q-R) EN SITUATION DE COMMUNICATION ASSISTÉE PAR UN(E) INTERPRÈTE}

\begin{abstract}
Apfelbaum Brigit, La gestion du couple QUESTION-RÉPONSE (Q-R) en situation de communication assistée par un(e) interprète. [The interactional management of question-answer sequences in interpreter-mediated discourse]. Studia Romanica Posnaniensia, Adam Mickiewicz University Press, Poznań, vol. XXV/XXVI: 2000, pp. 3-14, ISBN 83-232-0965-0, ISSN 0137-2475.

This paper explores the interactional management of question-answer sequences in interpreter-mediated business encounters and discusses didactic applications for interpreter training. A close analysis of transcripts from authentic audiotaped data reveals how the turn-taking system in the translational mode is particularized to contextual needs, i.e., as talk progresses, the business partners, rather than addressing one another, take turns being 'bystanders' as the other one communicates with the interpreter respectively. The interpreter takes and is given responsibilities to make the question-answer sequences work, especially as far as respecting conditional relevances is concerned. This system seems to be fully functional for a professional management of such situations and thus could be used as an analytical tool in training future liaison interpreters.
\end{abstract}

\section{INTRODUCTION}

Le couple QUESTION-RÉPONSE (Q-R) a constitué, depuis ses débuts, un objet privilégié de l'analyse conversationnelle d'orientation ethnométhodologique; il est considéré comme le format de base de toute sorte d'interaction verbale - aussi bien en situation informelle qu'en situation de communication plus formelle (cf. Sacks, Schegloff, Jefferson 1974) ${ }^{1}$. Dans le cadre de cette contribution, je me propose de

' Le couple Q-R a souvent été mentionné comme format prototypique de la paire adjacente (adjacency pair), celle-ci étant «un type plus ou moins fondamental d'organisation de la conversation» (Sacks, April 1972, p. 17, cité d'après Bange 1992). C'est le principe de dépendance conditionnelle (principal of conditional relevance) qui régit le fonctionnement des paires adjacentes, de manière que: «A une question, la réplique préférentielle est la réponse informative» (Bange 1992, p. 41). 
discuter des exemples de ce format provenant de situations d'interaction traduisante (cf. Müller 1989), c'est-à-dire de situations de communication où des interlocuteurs de langue différente recourent à des interprètes pour se (faire) comprendre. L'intérêt d'une telle analyse réside tout d'abord dans le fait que l'ordre séquentiel dans les couples Q-R semble particulièrement difficile à gérer dans l'interaction traduisante et que l'observation du travail conversationnel d'intercompréhension des participants qui en résulte peut nous donner accès à certaines particularités d'ordre plus général de ce type d'interaction. Pour prendre un exemple plus concret de la complexité des choses: lorsque des hommes d'affaire allemands p.ex. se font accompagner, lors d'une visite de la foire de Poznań, d'un(e) interprète qui veut bien les aider à communiquer avec des exposants polonais, ils sont généralement obligés de s'adresser, à chaque fois, à l'interprète avant que leurs partenaires potentiels puissent leur répondre. La même chose est valable pour les personnes voulant échanger des idées avec eux: elles doivent d'abord s'adresser à l'interprète avant que leurs réponses soient traduites vers l'allemand ${ }^{2}$.

Ce qui est intéressant d'un point de vue analytique, c'est alors de regarder de plus près comment les participants d'une interaction traduisante s'adaptent à cette contrainte de la double orientation en fonction du récepteur (recipient design) et quels sont les procédés qui leur permettent, dans un contexte particulier, de surmonter systématiquement les problèmes d'organisation de la prise de parole qui en résultent.

D'un point de vue traductologique et didactique, c'est évidemment le travail de l'interprète-médiateur qui est particulièrement intéressant à analyser. Vu le nombre croissant de situations (pas seulement en Europe) où nous faisons intervenir des interprètes-médiateurs dans notre vie professionnelle ou privée - je ne mentionne ici que les situations de formation bi- ou multilatérale, les négociations entre partenaires commerciaux, la communication avec des institutions comme les services sociaux, médicaux ou judiciaires lors d'un séjour à l'étranger - il semble urgent d'améliorer la formation des futurs médiateurs et de fonder leur formation sur des études empiriques dans ce domaine. Cela semble d'autant plus important que les professionnels de l'interprétation, mettant traditionnellement l'accent sur le principe de la neutralité,

Lorsqu'on essaie de caractériser linguistiquement ce qui permet aux interlocuteurs de se signaler le type de dépendance conditionnelle en question, on peut surtout retenir l'axe morpho-syntaxique (morphèmes interrogatifs, ordre des mots dans la QUESTION) et l'axe prosodique (intonation montante ou descendante dans la QUESTION; accentuation de certains éléments dans la QUESTION et dans la RÉPONSE). Par ailleurs, pour tenir compte des différents types de couples Q-R et de leur fréquence dans différents types de textes, il semble nécessaire d'intégrer également des aspects sémantiques (comme la demande de différents types d'information). C'est ainsi qu'on peut faire la différence entre le fonctionnement séquentiel des questions totales et celui des questions partielles, opposer les questions simples à des questions multiples, caractériser des questions alternatives ou rhétoriques, ou bien comprendre le rapport entre la fréquence d'un type de couple Q-R particulier et le type de texte construit, etc. (cf. Weinrich 1982, 1993; Léon 1997).

2 Pour l'analyse de situations d'interaction traduisante naturelles, cf. p.ex. Knapp, Knapp-Potthoff 1985 ou Müller 1989; pour l'interprétation en milieu juridique cf. p.ex. Scheffer 1997. 
ne sont pas toujours conscients de la complexité de leur tâche ou se considèrent bons interprètes seulement lorsqu'ils fonctionnent «comme des machines» et qu'ils «disparaissent» derrière les interactants primaires ${ }^{3}$.

Alors que, selon les modèles normatifs, ce seraient les interactants primaires qui attribueraient - grosso modo - chaque deuxième tour de l'échange aux interprètes et qui établiraient ainsi, au fur et à mesure, le format normal de l'interaction traduisante (cf. Knapp, Knapp-Potthoff 1985), l'analyse de différents corpus révèle que toutes sortes de procédés de co-sélection et de co-construction peuvent être mis en place par l'ensemble des participants, et cela évidemment non seulement dans des couples Q-R. Nous verrons alors que, pour gérer l'intercompréhension et la prise de la parole, les participants recourent, malgré une certaine «opacité» de la situation, à des méthodes qui ressemblent à celles qu'on trouve dans des échanges conversationnels sans médiateur linguistique. Tout en traduisant systématiquement les paroles des interactants primaires, les interprètes interviennent en permanence comme participants et font fonctionner le couple Q-R.

Pour illustrer ce phénomène, je me propose d'examiner une série d'exemples d'un corpus que j'ai pu enregistrer en accompagnant une interprète qui travaille régulièrement sur une grande foire industrielle en Allemagne. J'ai choisi plus particulièrement des extraits d'une situation où un visiteur espagnol essaie de contacter un exposant de luminaires italiens.

\section{LE CONTEXTE SITUATIONNEL DES DONNÉES}

Comme il vient d'être mentionné, mes données proviennent d'un contexte situationnel particulier, à savoir d'une première rencontre lors d'une foire industrielle allemande. C'est un visiteur espagnol (SP) qui veut se renseigner sur les possibilités de distribution des produits d'une firme italienne en Espagne et qui s'est adressé au service interprétariat (gratuit) de la foire où il a demandé «quelqu'un pour l'espagnol». Avant d'arriver au stand de la firme italienne, SP a expliqué à l'interprète (INT) quel type d'information il cherche à obtenir du représentant de cette firme. Sur place, c'est INT qui établit le contact entre les deux hommes d'affaires et négocie avec l'exposant de luminaires $(\mathrm{L})$, qui s'avère par ailleurs de nationalité néerlandaise; ce dernier va essayer de communiquer en allemand. Après cette ouverture gérée par INT, SP commence à poser des questions à L:

\footnotetext{
${ }^{3}$ Ainsi constate Gile (1995): «l'interprète de conférence est un médiateur 'transparent', qui se confond avec l'orateur (...)» (p. 146).

Qian (1994) affirme qu'un interprète professionnel éviterait toute contribution active de sa part: „A professional interpreter (...) would suppress such instincts or impulses to interact with the participants of a communication event, remain cool and maintain his role as an instrument in facilitating communication across linguistic and cultural borders" (p. 218).
} 
- tout d'abord, il essaie de savoir si la firme coopère déjà avec un concurrent espagnol (ce qui excluerait toute coopération et l'amènera finalement à reprendre sa carte de visite, échangée préalablement avec son partenaire potentiel),

- puis il essaie de comprendre des détails sur le type de produit distribué par la firme en question, avant de suggérer une suite raisonnable à cet entretien (échange de catalogues par la poste, etc.).

De l'autre côté, L, après avoir donné les informations que SP lui demande, pose quelques questions à son tour, ce qui lui permet probablement de transmettre le résultat de ces négociations à ses patrons.

La grande majorité des couples Q-R est alors déclenchée par des QUESTIONS qui présupposent déjà un certain savoir de la part de ceux qui «s'interrogent» - particularité sémantico-pragmatique qui semble renvoyer, pour emprunter la terminologie de Stephen Levinson (1992), au type d'activité prédominante dans cette situation de première prise de contact entre deux hommes d'affaires ${ }^{4}$.

\section{LE TRAVAIL D'ORGANISATION DE LA PAROLE: QUELQUES EXEMPLES DE CORPUS}

Quant au travail d'organisation de la parole, il devient visible, pendant la production de couples Q-R dans le mode de l'interaction traduisante, au moins à deux niveaux différents:

- d'une part, lors de la production successive des ORIGINAUX et de leurs INTERPRÉTATIONS, un travail d'intercompréhension «intime» entre l'interprète et l'interactant primaire respectifs semble permettre de gérer la construction et l'alternance des tours ${ }^{5}$; parallèlement, les participants semblent procěder à des changements d'orientation de leurs contributions en fonction de différents récepteurs possibles, ce qui permet de signaler le «changement de statut des interlocuteurs et la mobilité des rôles» (Malheiros-Poulet 1995, p. 152) dans une situation particulière de «trilogue en mouvement» (ibid.) (cf. chapitre 3.1 $)^{6}$;

- d'autre part, l'interprète semble recourir à des commentaires métacommunicatifs ou métadiscursifs pour résoudre des problèmes d'organisation de la parole qui résultent de l'adjacence différée entre le premier et le second élément du couple Q-R (cf. chapitre 3.2).

${ }^{4}$ La durée totale de la rencontre est de 15 minutes environ; elle comprend une dizaine de couples Q-R dont huit ont été numérotés et regroupés selon le statut séquentiel et socio-pragmatique de leurs constituants (QUESTION vs. RÉPONSE; ORIGINAL vs. INTERPRÉTATION). Pour l'analyse ne sont retenues ni les questions rhétoriques ni les sollicitations indirectes réalisées sous forme de question.

${ }^{5}$ Les traces de ce travail d'intercompréhension deviennent particulièrement «visibles» lorsque $\mathrm{L}$ manifeste des problèmes d'expression en allemand (voir infra).

${ }^{6}$ Wadensjô (1995) explique le fonctionnement de ce type d'alternance avec le concept de footing de Goffman (cf. Goffman 1979) et suggère que ces prises de responsabilité nous montrent une des compétences les plus importantes des interprètes de liaison. 


\subsection{LA CO-CONSTRUCTION DES ORIGINAUX ET DE LEURS INTERPRÉTATIONS}

Pour illustrer les phénomènes de co-construction et d'orientation en fonction des récepteurs présents lors de la production des éléments constituant le couple Q-R dans le mode de l'interaction traduisante, je souhaite d'abord présenter la première séquence déclenchée par SP. Dans les quatre constituants de la séquence (1. QUESTION'-ORIGINAL, 2. QUESTION-INTERPRÉTATION, 3. RÉPONSE-ORIGINAL, 4. RÉPONSE-INTERPRÉTATION), nous trouvons des traces d'un travail conversationnel de co-construction qui s'effectue entre les participants.

\subsubsection{FORMULER L'ORIGINAL DE LA QUESTION}

Lorsque SP prend la parole pour la première fois, il fait comme si $\mathrm{L}$ pouvait le comprendre et réagir directement à sa question, mais, petit à petit, il oriente sa question vers l'interprète - qui, de son côté, se positionne comme son récepteur primaire par l'emploi de signaux de feedback (en 8) et incite SP à formuler une question alternative et complexe:

(1) QUESTION 1: ORIGINAL (Q1/ORIG-SP) ${ }^{7}$

7 SP .. bien, e:h yo lo que quería es e:h ver si usted actualmente

8 . están exportando . . en España' [si] tienen algún cliente o varios' [y::] $\rightarrow \quad I N T \quad\left[\mathrm{mhm}^{\prime}\right] \quad[\mathrm{mhm}$ ']

9 SP y que si no es así que intención tienen ellos eh . . para una representación $\rightarrow 10$ INT para para para la representación $\mathrm{mhm}^{\prime}$.

SP commence doucement (. . bien, e:h, 7) et utilise une formule métacommunicative yo lo que quería es e: $h$ ver si pour s'adresser directement à L: usted (7). Déjà tout de suite après, $L$ et son entreprise deviennent la ou les personne(s) dont SP parle à INT (están exportando:, 8, tienen algún cliente o varios', 8, que intención tienen ellos, 9), c'est-à-dire que SP crée une situation de mini-dialogue avec INT dans laquelle L devient un bystander (cf. Goffman 1979). Ainsi, ce qui avait commencé comme une question indirecte adressée à l'allocutaire de langue différente est alors transformé en requête indirecte adressée à l'interprète.

\subsubsection{FOURNIR L'INTERPRÉTATION DE L'ORIGINAL}

Après avoir ratifié les derniers mots de SP (en 10), INT rend en allemand ce que SP veut savoir:

(2) QUESTION 1: INTERPRÉTATION (Q1/INT)

10 INT äh es geht darum ob Sie schon einen

11 Vertreter in Spanien habn' ob Sie nach Spanien verkaufen' oder. schon

12 eventuell mehrere Klienten in Spanien haben- und, wie das wäre im Falle $\mathrm{L}$ ä:h

13 daß Sie noch niemanden haben, und daß hier dann einer.

\footnotetext{
${ }^{7}$ Symboles de transcription $\mathrm{cf}$. annexe.
} 
Ici nous voyons un procédé relativement typique de la part de INT: Dans l'interprétation de la première question complexe, elle renvoie à la contribution de SP comme s'il fallait en faire une question indirecte äh es geht darum ob (10) et s'adresse directement à $\mathrm{L}$ et sa firme: Sie $(10,11,13)$. Les élements auxquels $\mathrm{L}$ est supposé répondre sont introduits par $o b(10,11)$ et wie das wäre im Falle daß $(12 / 13)$; les éléments alternatifs ou additifs de la liste sont connectés par oder $(11)$ et und $(12,13)$.

\subsubsection{FORMULER L'ORIGINAL DE LA RÉPONSE}

L, qui avait essayé de prendre la parole à la fin de la première sěrie d'éléments énumérés (cf. 12), attend finalement pour la prendre jusqu'à un moment où INT ne semble pas avoir signalé clairement une place transitionnelle (cf. absence de marqueur prosodique et syntaxique, en 13 ). L répond en allemand (=R1/ORIG-L), tout en signalant qu'il considère INT comme la destinataire à qui s'adressent les informations qu'il donne:

(3) RÉPONSE 1: ORIGINAL (R1/ORIG-L)

$13 \mathrm{~L}$

[se râcle la gorge] wir

14 haben noch keine: Verkauf in Spanien wir haben . (......?) einige Leute aber

15 noch nicht eine: eine richtige Verbreitung und zwar ein[e:: ä::h] (.....?) wir

$\rightarrow \quad I N T$

16 haben noch nicht den richtigen gefunden .

$\rightarrow$ INT

aha-

[mhm]

Ici, comme dans pratiquement toutes les autres réponses qu'il donne, $\mathrm{L}$ formule les informations comme si c'était INT qui les lui avait demandées (wir haben noch keine..., 13/14; wir haben noch nicht..., 15/16). Au moment où il manifeste des difficultés pour trouver les mots (en 15), INT se positionne verbalement comme son destinataire avec un élément de feedback. Avant de se mettre à traduire, elle ratifie la réponse qu'il a donnée (aha-, 16).

\subsubsection{FOURNIR L'INTERPRÉTATION DE LA RÉPONSE}

Pour fournir l'interprétation de la première réponse (= R1/INT), INT passe à l'espagnol:

(4) RÉPONSE 1: INTERPRÉTATION (R1/INT)

16 INT

äh todavía no ha encontrado una

17 persona adecuada' äh. todavía no venden en España o sea todavía no

$\rightarrow \quad$ SP sí-

18 han encontrado y por eso no lo tienen,

Ce passage atteste également un procédé typique de la part de INT: en se référant à $L$ et sa firme à la troisième personne (no ha encontrado, 16; no venden, 17; no han 
encontrado, no lo tienen, 17/18), elle rapporte à SP ce que L lui a donné comme information. Comme SP, de son côté, se positionne comme le destinataire direct de INT(sí, 17), c'est L qui devient, pour reprendre la terminologie de Goffman, le bystander.

\subsubsection{RÉSUMÉ}

Alors que SP s'était adressé, dans sa première QUESTION, directement à L et qu'il avait seulement modifié son comportement en fonction de INT qui s'était positionnée comme son alliée locale (cf. Malheiros-Poulet 1995), les deux interactants primaires ne s'adressent, dans la suite de l'entretien, pratiquement plus directement l'un à l'autre. C'est dans des échanges respectifs avec l'interprète qu'ils font progresser le déroulement séquentiel des couples Q-R, et le footing de rapporteur (cf. Wadensjö 1995) signalé par l'interprète devient plus stable ${ }^{8}$.

${ }^{8}$ Pour donner un exemple d'une question que L pose à SP, je voudrais citer le couple Q-R 10 de mon corpus:

(5) Q10/ORIG-L

$\rightarrow 193 \mathrm{~L}$

194

(...) was was was

INT

macht der . Herr .. in Spanien, ist er eine ein Großhandel' ist er eine

195 mhm'

Agent oder ist das äh . eine ein Hersteller' oder-

INT

mhm'

Ici, L pose une question partielle directe à INT et renvoie explicitement à SP à la troisième personne: was was was macht der. Herr . . in Spanien. (193/194), ist er... (193-195). INT, de son côté, produit des éléments de feedback $(194,195)$; le résultat en est la constitution d'une question complexe et alternative dont L signale le statut par oder (194, 195).

Lorsque INT se met à traduire la question (Q10/INT), elle dirige sa question directement à SP:

(6) Q10/INT

$\rightarrow 195 \quad I N T$

196

representante o distribuidor,

usted es productor'o

$\rightarrow \quad$ SP

representante,

Parallèlement à son interprétation, SP ratifie déjà un élément que INT a mentionné (representante, 196) et produit ainsi un élément de réponse anticipé qu'il poursuit en R10/ORIG-SP (sí, sí, yo tengo una empresa es . es una comercial, 196/197):

(7) R10/ORIG-SP

196

SP
.) represen/tante,

representante, [sí, sí, yo tengo una empresa es . es una

197 SP comercial,

Lorsque SP signale prosodiquement et syntaxiquement une place transitionnelle (en 197), INT se met à traduire pour L (RIO/INT), tout en renvoyant à la troisième personne à SP cette fois-ci (er ist..., 197):

(8) RIO/NT

$\rightarrow 197$ NT ah ja er ist Repräsentant . für eine . ja das is son Di (...?) so ein

198

Verteilernetz also ja'

$\rightarrow \quad$ L mhm- ja,

Vers la fin de l'interprétation fournie par INT, nous remarquons encore une autre particularité: lorsque des problèmes de formulation deviennent manifestes lors de la recherche d'un équivalent allemand pour le terme espagnol comercial (cf. hésitations et commentaires métadiscursifs en 197-198), L participe à cette recherche lexicale du moins de façon minimale et signale ainsi avoir compris ce que INT vient de dire. 


\subsection{LE TRAVAIL DE RÉPARATION SUR LES DÉPENDANCES CONDITIONNELLES}

Au-delà du travail de compréhension du contenu, on trouve, dans les échanges entre INT et les interlocuteurs primaires, des traces d'un travail de réparation sur les dépendances conditionnelles. Dans ces cas, INT intervient de façon métacommunicative pour préciser l'acte illocutoire en construction - soit en signalant à un interlocuteur ce qu'elle va «faire» de son ORIGINAL, soit en précisant ce qu'elle a retenu comme dépendance conditionnelle établie par l'autre interactant primaire.

Regardons d'abord un extrait où INT semble intervenir parce qu'il n'est pas clair quel type de contribution SP veut faire et quelle dépendance conditionnelle il s'agit d'établir:

(9) Q5/ORIG-SP

103 SP

pero . para ellos . lo importante es

104 lo que ha dicho, de . de hacer el montaje allí, en España, tendrán que enviar

INT äh

105 el material' despiezado' para montarlo hacer el ensemblaje allí en España,

$106 \quad$ eso es, o es una

$\rightarrow \quad$ INT äh. voy a preguntar si eso es el foco- ya . o sí. pueden hacerlo la 107 posibilidad, eso es, INT mhm-

Dans cet extrait, nous voyons que INT écoute d'abord ce que SP expose puis essaie de prendre la parole à la première place transitionnelle (cf. complétude syntaxique): äh (104). Jusque là, SP n'a pas signalé morpho-syntaxiquement qu'il veut poser une QUESTION et ne le fait toujours pas quand INT lui rend la parole. Ce que SP dit peut être compris comme une reformulation de ce que $\mathrm{L}$ vient de dire (lo que ha dicho, 104); le seul signal qui puisse être compris comme indice de QUESTION, c'est l'intonation montante (el matériel' despiezado', 105). Lorsque INT prend la parole à la prochaine place transitionnelle, elle se réfère à ce que SP vient de dire et suggère l'acte illocutoire qu'elle a l'intention d'en faire äh. voy a preguntar si eso es el foco- (106). SP ratifie aussitôt (eso es, 106) et les deux interlocuteurs se mettent à clarifier davantage ce que INT doit transmettre à L (INT: o sí pueden hacerlo la, 106; SP: o es una posibilidad, eso es, 106-107).

Dans d'autres cas, INT intervient plus tard dans la séquence et rappelle la dépendance conditionnelle établie par une QUESTION à un moment où un interactant primaire est déjà en train de produire une RÉPONSE, c'est-à-dire un nouvel ORIGINAL pour elle. Dans ce cas, le travail de réparation devient beaucoup plus complexe parce que l'interprète doit d'abord reformuler (une partie de) la question et qu'ensuite l'interactant primaire actuel est obligé de reformuler la (partie de) réponse qu'il avait commencé à donner. Regardons les trois extraits suivants (R4/ORIGa-L; R/Q4-INTb; R4/ORIGb-L); 
(10) R4/ORIGa-L

$86 \mathrm{~L}$

. . ja was was wir

87 INT suchen ist . eine:: (Kontakt?) oder eine eine Agent für die längere Zeit88 wann wir . heut ein Agent machen, und nach dri Monate sagen . ja . INT $j a{ }^{\prime}$

89 Entschuldigung. Sie sind nicht richtig' dann sind wir falsINT ja' $\mathrm{mhm}$ -

(11) R/Q4/INTb

nein es is

90 auch nein die Frage is noch anders, so wie ich sie verstanden habedie Frage
$\mathrm{L}$
also
ah
okay-

91 is ob Sie jetz sagn wir mal sofort jemanden suchen oder erst in einem Jahr

92 jemanden suchen- so hatte ich das verstanden,

(12) R4/ORIGb-L

$92 \mathrm{~L}$

93

$\rightarrow \quad I N T$

94

$\rightarrow \quad I N T$

95

richtige Mann da kommt dann::

ja wann: . ja . wann die okay dann sofort, dann dann dann gehen wir-

äh äh ein: das ist nicht äh heute ja vielleicht . äh . in drei Monaten ja-ja, perfecto, ja, (bißchen weniger) das ist . das Kontakt was wir da auf der Messe haben und

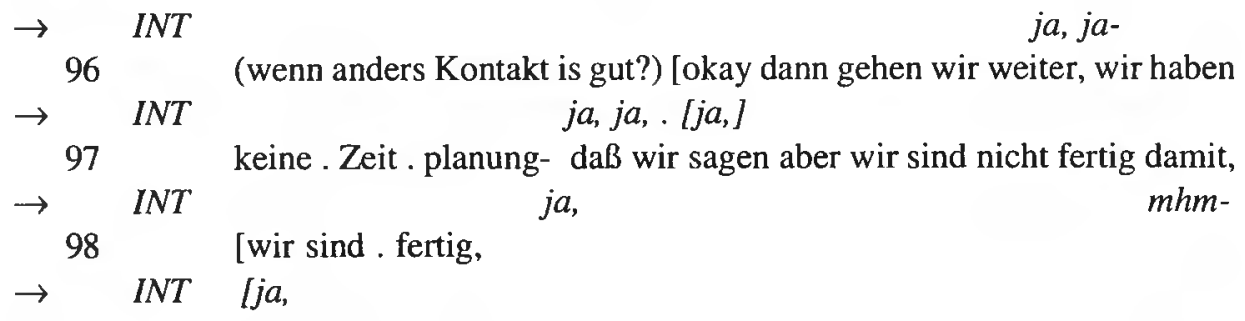

Dans un premier temps, INT écoute ce que L formule comme réponse (86-89), mais - au lieu de se mettre à traduire - l'interrompt avec un commentaire métadiscursif et une reformulation de «sa» version de la question: nein es is auch nein die Frage is noch anders, so wie ich sie verstanden habe- die Frage is ob Sie jetz sagn wir mal sofort jemanden suchen oder erst in einem Jahr jemanden suchen- so hatte ich das verstanden, (89-92).

Après cela, L, qui accepte l'intervention de INT (also..., ah..., okay-, 90), se reprend et INT co-construit cette reprise (cf. achèvement interactif: okay dann sofort, 93; feedback: ja-ja, perfecto, etc., 94 sq.), tout en signalant à L que l'adjacence entre le premier et le deuxième élément du couple $\mathrm{Q}-\mathrm{R}$ fonctionne mieux maintenant. 
Pour me résumer: Que ce soit en précisant quel type de dépendance conditionnelle un interactant primaire veut créer pour son destinataire ou bien que ce soit en intervenant avec une correction métacommunicative lorsque le destinataire d'une question n'a pas respecté la dépendance conditionnelle établie - l'interprète semble savoir réparer les problèmes d'organisation en recourant à son savoir sur les particularités du type d'activité en construction.

\section{CONCLUSION}

En guise de conclusion, je voudrais d'abord résumer mes résultats en discutant ce qu'on peut en retenir pour une meilleure compréhension des situations d'interaction traduisante, puis suggérer quelques applications didactiques.

Tout d'abord, nous avons pu voir que la constitution des couples Q-R avec ses constituants QUESTION et RÉPONSE demande un travail conversationnel d'intercompréhension particulier de la part des participants lorsque ceux-ci communiquent dans le mode de l'interaction traduisante; les procédés mis en place lors de la production des ORIGINAUX et des INTERPRÉTATIONS semblent faciliter la compréhension et l'expression des segments à traduire. Nous aurions donc ici des traces de ce que des traductologues réclament depuis longtemps comme fondamental dans le processus de traduction orale (cf. Seleskovitch, Lederer 1993).

De plus, les changements de positionnement de l'interprète ainsi que l'alternance entre le discours indirect et les échanges directs avec les interactants primaires semblent être fonctionnels pour la gestion de la situation; ils permettent de signaler localement quelles sont les responsabilités assumées par l'interprète veillant à faire fonctionner la communication entre participants de langue différente ${ }^{9}$.

Pour ce qui est du recours aux commentaires métadiscursifs et métacommunicatifs, il semble surtout permettre à l'interprète de réparer les problèmes d'organisation de la parole liés à «l'adjacence différée» des éléments constituant le couple Q-R. L'interprète, qui partage un savoir contextuel avec les interactants primaires, semble utiliser ce savoir sur le type d'acticité en construction pour intervenir de manière à faciliter la prise de contact entre les deux hommes d'affaires. Nous avons pu observer des tendances similaires dans d'autres situations de discours médiateur (cf. Apfelbaum / Berkenbusch sous presse), et cela non seulement lorsqu'il s'agissait d'intermédiaires-interprètes mais aussi lorsque des animateurs de radio servaient d'intermédiaires ${ }^{10}$ entre différents auditeurs d'une station de radio.

${ }^{9}$ Wadensjo (1995) suggère que ces prises de responsabilité nous montrent une des compétences les plus importantes des interprètes de liaison.

${ }^{10}$ Pour le terme d'intermédiaire linguistique voir aussi Malheiros-Poulet (1995). 
Du point de vue de la didactique de l'interprétation de liaison, il paraît très fructueux d'attirer l'attention des futurs interprètes sur ces aspects de leur profession. Dans le cadre d'un mini-curriculum intitulé interprétation de laison technique («Fach- und Verhandlungsdolmetschen»), cet aspect a été développé ces dernières années à l'université de Hildesheim. D'une part des travaux dirigés sont organisés où deux enseignants de langue différente simulent des situations de communication avec les étudiants servant d'interprètes, et, d'autre part, dans le cadre d'un séminaire de linguistique appliquée, des enregistrements (audio et vidéo) de ce type de situation sont analysés avec les étudiants.

De la même manière que la didactique des langues étrangères a jugé utiles les résultats des travaux sur la communication exolingue et a essayé d'intégrer ces résultats dans son domaine, les «outils conversationnels» s'avèrent à mon avis également prometteur pour le développement de nouveaux concepts en didactique de l'interprétation de liaison.

\section{ANNEXE}

Principaux symboles de transcription

\begin{tabular}{ll}
\hline & $\begin{array}{l}\text { interruption très courte dans un énoncé ou entre les énoncés de deux interlocuteurs } \\
\text { courte pause } \\
\text { pause moyenne } \\
\text { enchaînement rapide }\end{array}$ \\
\hline & $\begin{array}{l}\text { intonation montante } \\
\text { intonation descendante } \\
\text { intonation en suspens }\end{array}$ \\
\hline
\end{tabular}

que: a:::h wann: allongement d'un mot/d'une syllabe/d'un son

nicht $(t) \quad$ articulation relâchée

(Kontakt?) (partie d'un) énoncé incertain

(.......?) (partie d'un) énoncé incompréhensible

[bas $]+\quad$ description du mode d'articulation, du comportement non-verbal; placée devant ou au-dessous de l'énoncé respectif et valable jusqu'au «+»

$[[$ surpris $]]+\quad$ interprétation que le transcripteur signale comme subjective;

précède l'énoncé qu'il caractérise, valable jusqu'au signe «t»

L [ok]ay

chevauchement de deux ou plusieurs énoncés; les contributions de l'interprète

INT ja,]

(INT) sont toujours mises en italique 


\section{BIBLIOGRAPHIE}

A pfelbaum, B. (en préparation), I think I have to translate first... Zu Problemen der Gesprächsorganisation in Dolmetschsituationen sovie zu einigen interaktiven Verfahren ihrer Bearbeitung. In: Apfelbaum, B., Müller, H. (éds), Fremde im Gespräch.

A pfelbaum, B., Berkenbusch, G. (sous presse), L'organisation textuelle en discours médiateur: Comment procèdent les animateurs de radio et les interprètes. Actes du $\mathrm{XXI}^{e}$ Congrès International de Linguistique et Philologie Romanes, Palermo, 18 - 24 septembre 1995.

B ange, P. (1992), Analyse conversationnelle et théorie de l'action, Paris: Didier.

Gile, D. (1995), Regards sur la recherche en interprétation de conférence, Lille: Presses Universitaires de Lille.

Goffman, E. (1979), Footing, Semiotica 25, 1-29.

$\mathrm{K} n$ app, K., Kn app-Potth of f, A. (1985), Sprachmittlertätigkeit in der interkulturellen Kommunikation. In: Rehbein, J. (éd.), Interkulturelle Kommunikation, Tübingen: Narr, pp. 450-463.

Léon, J. (1.997), Approche séquentielle d'un objet sémantico-pragmatique: le couple Q-R, questions altematives et questions rhétoriques. Revue de Sémantique et Pragmatique 1, pp. 23-50.

Levins on, S. C. (1992), Activity types and language. In: Drew, P., Heritage, J. (éds.), Talk at Work. Interaction in Institutional Settings, Cambridge: Cambridge University Press, pp. 66100.

Malheiros-Poulet, M. E. (1995), Le rôle de l'intermédiaire linguistique dans les situations de contact interculturels. In: Kerbrat-Orecchioni, C., Plantin, Ch. (éds.), Le trilogue, Lyon: Presses Universitaires de Lyon, pp. 134-160.

Mülle r, F. (1989), Traduction en conversation bilingue. Quelques aspects de son ordre séquentiel et interactif. In: Minorisation linguistique et interaction. Actes du symposium organisé par l'association internationale de linguistique appliquée et la commission interuniversitaire suisse de linguistique appliquée, Neuchâtel, 16-18 spt. 1987. Textes réunis par Bernard Py et René Jeanneret, Université de Neuchâtel, pp. 59-72.

Qian, Hu (1994), Looking at Interpretation from a Communicative Perspective, Babel 40/4, pp. 214-221.

Sacks, H., Scheg lo ff, E. A., Jefferso n, G. (1974), A simplest systematics for the organization of turn-taking for conversation, Language 50, pp. 696-735 (aussi dans: Schenkein, J. (éd.) (1978), Studies in the Organization of Conversational Interaction, New York: Academic Press, pp. 7-56).

Scheffer, Th. (1997), Dolmetschen als Darstellungsproblem. Eine ethnographische Studie zur Rolle der Dolmetscher in Asylanhörungen, Zeitschrift für Soziologie 26/3, pp. 159-180.

Seleskovitch, D., Lederer, M. (1993), Interprêter pour traduire. Paris, Didier Erudition.

Waden s jö, C. (1995), Dialogue interpreting and the distribution of responsibility, Hermes, Journal of Linguistics 14, pp. $111-129$.

Weinrich, H. (1982), Textgrammatik der französischen Sprache, Stuttgart: Klett.

Weinrich, H. (1993), Textgrammatik der deutschen Sprache, Mannheim: Dudenverlag. 